\title{
RFID-based Object-Centric Data Management Framework for Smart Manufacturing Applications
}

DOI:

10.1109/JIOT.2018.2873426

\section{Document Version}

Accepted author manuscript

Link to publication record in Manchester Research Explorer

\section{Citation for published version (APA):}

Meng, Z., Wu, Z., \& Gray, J. (2018). RFID-based Object-Centric Data Management Framework for Smart Manufacturing Applications. IEEE Internet of Things Journal . https://doi.org/10.1109/JIOT.2018.2873426

\section{Published in:}

IEEE Internet of Things Journal

\section{Citing this paper}

Please note that where the full-text provided on Manchester Research Explorer is the Author Accepted Manuscript or Proof version this may differ from the final Published version. If citing, it is advised that you check and use the publisher's definitive version.

\section{General rights}

Copyright and moral rights for the publications made accessible in the Research Explorer are retained by the authors and/or other copyright owners and it is a condition of accessing publications that users recognise and abide by the legal requirements associated with these rights.

\section{Takedown policy}

If you believe that this document breaches copyright please refer to the University of Manchester's Takedown Procedures [http://man.ac.uk/04Y6Bo] or contact uml.scholarlycommunications@manchester.ac.uk providing relevant details, so we can investigate your claim.

\section{OPEN ACCESS}




\title{
RFID-based Object-Centric Data Management Framework for Smart Manufacturing Applications
}

\author{
Zhaozong Meng, Member, IEEE, Zhipeng Wu, Senior Member, IEEE, John Gray
}

\begin{abstract}
The Internet-of-Things (IoT) empowered technical revolution in manufacturing industry allows pervasive sensing and ubiquitous data access through the lifecycle of products. Although great efforts have been devoted to bridge the gap between industrial operations and information technologies, it still faces technical challenges to unobtrusively monitor the entire lifecycle of products via a boundary-less information flow for Product Lifecycle Management (PLM) in highly adaptive manufacturing. This investigation presents an IoT sensing and networking framework for seamless data integration and ubiquitous access in smart manufacturing, focusing on product identification, data modeling, inter-phase data integration and ubiquitous data access. The highlights of this investigation are: (1) Radio Frequency Identification (RFID) and virtual Universal Unique Identifier (UUID) dual identifier online item-specific data integration, (2) RFID based online product object localization and unique identification, (3) object-centric manufacturing process modeling for inter-phase data integration, and (4) RFID/QR code encoding method for ubiquitous data sharing between product trading lifecycle phases. Finally, the implementation of presented methods in EU PickNPack food manufacturing production line is reported, and the practice has proved the feasibility and advantages.
\end{abstract}

Index Terms-Smart manufacturing, IoT, PLM, UUID, RFID.

\section{INTRODUCTION}

$\mathrm{T}$ he increasing convergence of IoT technologies with the manufacturing industry has created new opportunities for smart manufacturing [1-3]. The pervasive sensing techniques provide means for comprehensive monitoring of industrial operations, and ubiquitous Machine-to-Machine (M2M) communications leverage collaborative automations between machines [4]. This new industrial computing paradigm featuring pervasive sensing, ubiquitous data access, intermachine understanding, data analysis and optimization has gained interests in both industry and academia, which is marked with Industrial IoT (IIoT), Industry 4.0, Industrial Internet, Smart Manufacturing, Cyber-Physical Systems (CPS), etc. [5,6]

This IoT-enabled new computing paradigm can potentially combine the raw material supply, production operations and product trading, and forge a closed Product Data Management (PDM) loop, where information from any phase is recorded to affect processes and decision-making in other phases. The interoperability introduced by IoT technologies therefore promise a technical solution which could streamline the flow of information about products and related processes throughout the products' lifecycles such that the right information in the right context at the right time can be made available [7].

Copyright (c) 2012 IEEE. Personal use of this material is permitted. However, permission to use this material for any other purposes must be obtained from the IEEE by sending a request to pubspermissions@iee.org.
Therefore, the concerned entities could improve production development at all phases of product lifecycle. The collected data can be used for further analysis and the analytical insights can be applied to optimize the products and production processes [8]. The concept of Closed-Loop PLM (CLPLM) could benefit the stakeholders in the manufacturing, including raw material suppliers, manufacturers, logistics and supply chain. A wide spectrum of sensor technologies and Information and Communication Technologies (ICT) in the IoT scope are applied to revolutionize the manufacturing industry with focus on sensor networks, M2M communications, production process modeling, Manufacturing Reference Architectures (MRA), and data analytics $[9,10]$. A number of heuristic investigations are conducted to optimize the sensing, communication, and data management at network, software and system levels.

Since PLM combines the industrial processes through the product lifecycle in IoT-enabled manufacturing, each 'Thing' including raw materials, machine parts, product components, and finished products are expected to be uniquely identifiable by the functional machines and the data is available to all interested machines in the information framework [11]. However, traditional process-centric data management solutions are constrained by automatic and unobtrusive identification techniques and strategies for automatic operations when identification labels are not applied. It faces huge technical challenge to uniquely identify products through their entire lifecycles and provide a complete information flow between machines, sectors and enterprises [12]. In other words, a seamless information integration context for smart manufacturing is not technically ready.

This investigation aims to determine the underlying challenges for lifecycle data integration and sharing in multiplephase smart manufacturing, bridge the technological gap with IoT techniques and object-centric methods, and gain system level interoperability for seamless and efficient data integration through the lifecycle of products. For this purpose, this investigation combines the efforts of the following aspects: (1) RFID and virtual UUID dual identifier for online item-specific product data integration; (2) RFID based online product localization and identification for seamless data integration; (3) object-centric manufacturing process model for inter-phase data integration; and (4) RFID/QR code encoding method for ubiquitous data sharing between product trading phases. Finally, the implementation of the presented methods in PickNPack food manufacturing line is reported, and the practice proves the feasibility and advantages.

The remainder of this article is structured as follows: A review of the enabling technologies and related studies are introduced in Section II. The IoT-based PLM framework and the corresponding technical solutions are presented in Section III. Then, the implementation of the proposed solutions in PickNPack food manufacturing line is presented in Section IV. Finally, discussions are given in Section V and conclusions are drawn in Section VI. 


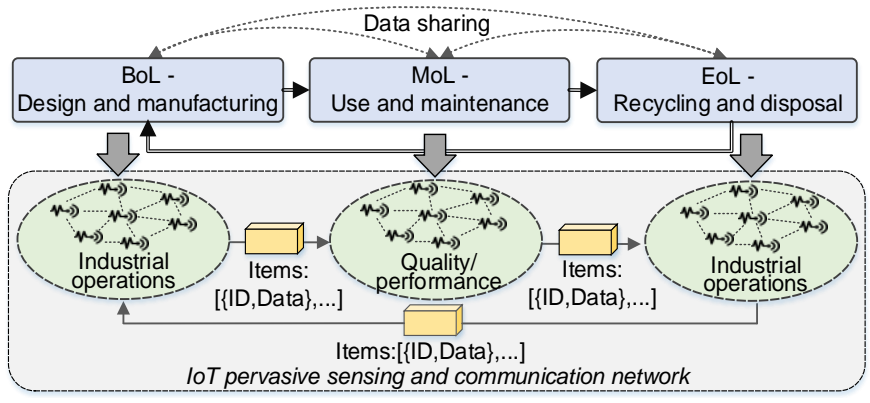

Fig. 1. PLM in IoT-based Manufacturing

\section{PLM IN IOT-BASED SMART MANUFACTURING}

To gain the system level interoperability between all entities producing, storing, and consuming production information in a manufacturing system, a wide spectrum of enabling technologies is involved. This section briefly introduces the PLM process in IoT-enabled manufacturing industry, and then illustrates the building blocks and some related investigations.

\section{A. PLM in IoT-based Manufacturing}

With IoT technologies, the physical devices, material and product items in manufacturing, logistics, and supply chain are expected to be uniquely identified and the corresponding data be integrated and shared in an open information framework.

As shown in Fig. 1, with the pervasive sensing technologies, network infrastructure and data sharing architecture, the manufacturing phases such as design and manufacturing as the Beginning-of-Life (BoL), use and maintenance as the Middleof-Life (MoL), and recycling and disposal as the End-of-Life (EoL) can be observed and the collected data can be stored in a data repository $[11,13]$. The collected data can be used in real time for online decision-making in manufacturing, or for authorized business partners to access the data to optimize their products and production procedures.

\section{B. Building Blocks for PLM in IoT-based Manufacturing}

IoT-based manufacturing is usually supported by multiple levels of applied technologies, including hardware, software, communications, service platforms, and data science. With respect to IoT-enabled manufacturing industry, the building blocks can be classified into four levels: pervasive sensing techniques, machine connectivity and messaging, production management architecture, data analysis and optimization. The interests of this investigation are the former three levels of technologies to build the information framework for production monitoring, data integration and interoperable data sharing.

\section{1) Pervasive sensing techniques}

The pervasive sensing techniques are the key building blocks for IoT applications. The sensors and embedded electronics interface the physical world with information systems by sensing and monitoring the physical variables and operations.

In order to seamlessly integrate the data produced through the production process, some sensory technologies are applied to identify the products and connect the industrial processes. The mainstream product identification techniques are RFID, $\mathrm{QR}$ code and Barcode. According to the features in information capacity, reading speed, reading distance, directivity, multiple reading, reusability, security, and cost, etc., RFID outperforms visual identification solutions, especially in none-line-of-sight reading. In addition, mechanical encoder and laser trigger are also widely used for the synchronization of mechanical operations. In addition, RFID also offers the functionality of environment or product quality sensing by coupling sensors to RFID tracking [14]. Since the sensing technologies build the intermediary interfaces between physical operations and information systems, they play a critical role in the seamless data integration of IoT-based smart manufacturing systems.

2) Machine connectivity and messaging

The machine connectivity and networking technologies build the connections between functional machines, which promise optimized execution of manufacturing strategies by enabling interactive collaborations between machines.

Flexible machine presence and discovery, ubiquitous M2M communication, and inter-machine understanding are underlying enablers to take advantage of the sensing techniques and put highly adaptive manufacturing into practice. In order to gain the flexibility of interactions between connected machines, many M2M messaging protocols, middleware, and APIs are developed by Standard Development Organizations (SDOs) [15], such as Message Queuing Telemetry Transport (MQTT), Constrained Application Protocol (CoAP), Advanced Messaging Queue Protocol (AMQP), Extensible Messaging and Presence Protocol (XMPP), Data Distribution Service (DDS), Zero-MQ (ZMQ), MTConnect, and Open Platform Communications-Unified Architecture (OPC-UA). They are based on different technologies and for different applications scenarios. The selection of M2M messaging techniques should be based on the in-depth understanding of the messaging technologies and the object IoT system.

\section{3) Manufacturing management service architecture}

IoT-based business models and Manufacturing Reference Architectures (MRAs) based on Service Oriented Architecture (SOA), RESTful and Cloud Computing are also a key support for manufacturing management [16]. An appropriate service architecture can potentially benefit manufacturing systems in resource scheduling, optimal machine collaborations, manufacturing strategy optimization, and exploration of the capability of manufacturing systems. The exemplar MRAs are ETSI M2M service architecture, SMLC smart manufacturing platform, OneM2M harmonized reference architecture, and Microsoft DiRA, etc. [11] The common targets of the MRAs are openness, interoperability, inter-module collaborations and optimization of the information flow and industrial operations.

The techniques for product object identification, the models for inter-phase data integration and methods for ubiquitous data access are regarded as the key parameters of PLM in smart manufacturing. The integration of the IoT techniques with the manufacturing industry and the above-mentioned enabling techniques have provided sufficient technical support.

\section{Related Work}

PLM is a concept with multiple interpretations, and the most common definitions seem to be about how to manage information related to the design and manufacturing of products and their variations, which represents a rather computer-aided technologies oriented view of PLM [17]. The further interpretations like sustainable PLM and intelligent products attempt to extend PLM also to the usage, refurbishing, disposal and other lifecycle phases that product instances go through 
[18]. From conceptual and functional points of view, [7] describes PLM as a knowledge management system which supports the entire product value chain, which integrates people, information and processes across the extended enterprise through a common body of knowledge. PLM closes different knowledge loops throughout product lifecycle by enabling reuse of lifecycle knowledge in the development phase. The definitions reflect that the manufacturing industry tends to see PLM beyond traditional PDM in today's digital manufacturing. This is due to IoT techniques which establish a set of tools and technologies that provide a shared platform for collaborations among product stakeholders and streamline the flow of information along all phases of product lifecycle.

Uniquely identifying the product objects through their lifecycles is a critical step for PLM. The requirements of unique identifier of products for PLM is highlighted in [19] with analysis and comparison of the main approaches including EPC network, DIALOG, and WWAI. An RFID based framework for Product Lifecycle Information Management (PLIM) is presented with case studies to support decision making in different lifecycle phases of automotive industry [13]. In order to improve the flexibility of manufacturing, an Object-Oriented RFID (OORFID) mechanism is proposed by taking advantages of the efficient utilization of RFID memory, and is introduced into IoT manufacturing systems [20].

Innovations in system architecture and communication protocols with emerging ICTs to deal with the heterogeneity of technologies and complexity of systems is a widely investigated topic. The lack of generic and standardized interfaces for creating the needed information between devices is highlighted in [11], and the proposed design principles and a Quantum Lifecycle Management (QLM) interface standard is presented to fulfill the requirements. An M2M messaging mechanism for ubiquitous data access and event notification of IIoT applications is presented with experimental studies in [21], which showed certain flexibility in machine presence, discovery and ubiquitous M2M messaging for collaborative automations. With respect to software, a flexible information model and configurable software platform for IoT-based PLM is presented in [22] to cover the whole product lifecycle in order to integrate heterogeneous and distributed product information within and across organizations. To cover the full range of the products' lifecycle, a typology of standards relevant to PLM support that addresses the hierarchy of existing and evolving standards and their usage, and a suite of standards to support the exchange of product, process, operations and supply chain information are identified in [23]. In addition, a product information-modeling framework is described in [24] to support full range of PLM information needs based on the Core Product Model (CPM), Open Assembly Model (OAM), Design-Analysis Integration Model (DAIM) and Product Family Evolution Model (PFEM). These abstract models are with specific semantics about a particular domain to be embedded within the usage of the models for the domain.

According to the related work, the concept of PLM and its benefits in combining all lifecycle phases of manufacturing for product development has attracted interests in many studies. Due to the complexity of product lifecycle processes and communication barriers, the unique identification and unified management of distributed and heterogeneous product data that cover all the lifecycle phases are still challenging tasks. Most studies focus on the system architecture design and data modeling for supply chain data management [25]. It lacks indepth investigations to design from a system perspective to bridge the gap between the on-manufacturing-line mechanical operations and the information technologies. Therefore, to build a loose coupled interoperable information flows between concerned entities with IoT sensing and messaging techniques covering the entire lifecycle of products especially onmanufacturing-line procedures and inter-phase connects is a critical task for PLM in today's manufacturing industry.

\section{Problem Statement}

Product data collection and management has been widely used in the manufacturing industry. However, the challenges facing IoT-enabled highly adaptive manufacturing systems to uniquely identify the whole lifecycle of products, especially for pre-label unmanned automations, and provide a through lifecycle boundary-less information flow have not been adequately addressed. The related investigations focus on the information modeling, while critical techniques and systematic strategies have not been reported. The major technical challenges are: (1) item-specific identification and data integration of on-manufacturing-line phases and offline logistics phases, (2) online real-time moving object identification and localization for data integration of highly adaptive operations, (3) manufacturing process modeling for inter-phase data integration and sharing between trading partners, and (4) efficient and flexible data dissemination for ubiquitous data sharing in the trading phases.

\section{E. The Scope of this Investigation}

This investigation aims to gain system level interoperability in smart manufacturing for PLM by taking advantage of IoT sensing and networking techniques and object-centric methods, focusing on lifecycle product identification, process modeling, data integration and sharing. The techniques for product identification to combine the lifecycle phases, the manufacturing process models for inter-phase data integration, and the data dissemination methods for data sharing are the focal research topics. The proposed solutions are implemented in PickNPack food manufacturing line for online data integration, ubiquitous data access for machine collaborations, and inter-phase data integration between source material supplier, manufacturer and product supply chain. The practice of IoT-enabled product lifecycle data integration and ubiquitous data access in PickNPack manufacturing line is reported to prove the feasibility of the proposed technical solutions.

\section{IOT-BASED PLM FRAMEWORK}

To deal with the addressed technical issues, this section describes a technical framework for PLM of IoT-based manufacturing. The corresponding technical solutions for product identification and data integration in IoT-enabled highly adaptive manufacturing context are illustrated.

\section{A. IoT-based PLM System Architecture}

The mission of this system architecture is to provide a platform to integrate the real-world lifecycle phases in a data 


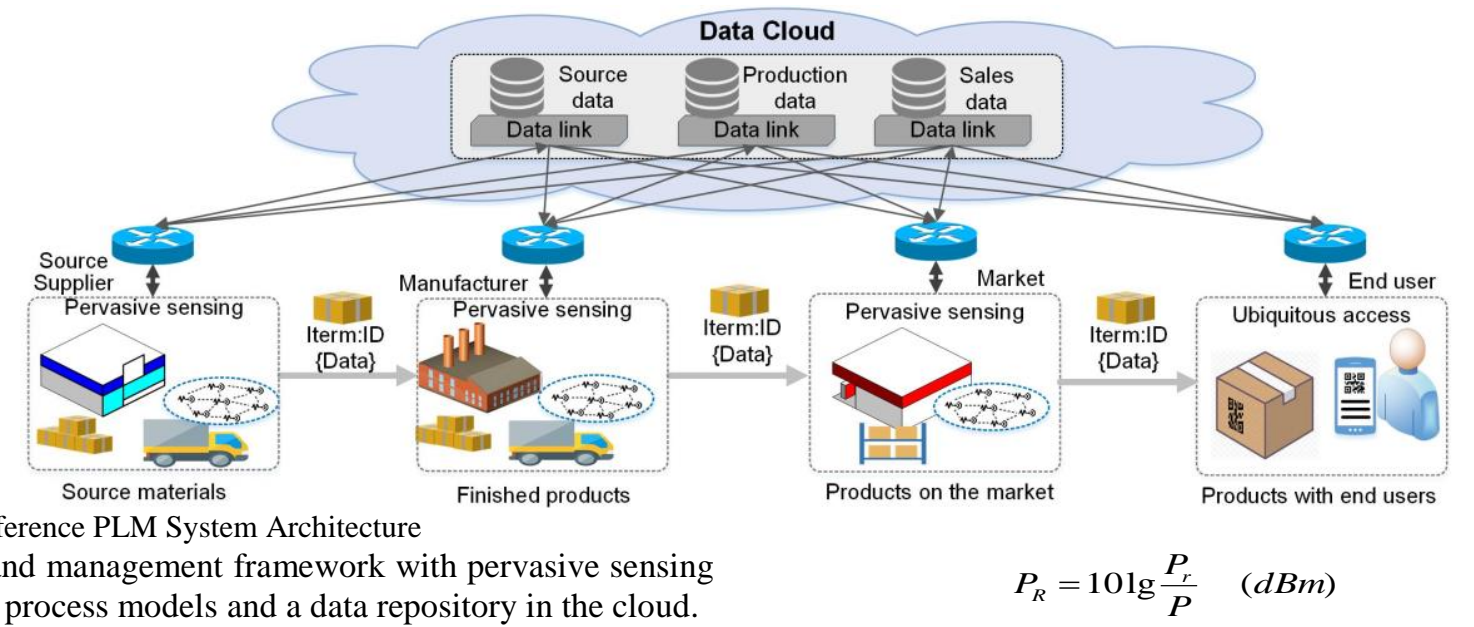

Fig. 2. A Reference PLM System Architecture collection and management framework with pervasive sensing techniques, process models and a data repository in the cloud.

To this end, a suggested cloud based system architecture is as shown in Fig. 2. With IoT pervasive sensing technologies such as RFID, QR code and barcode, the product objects can be labeled and identified with their IDs. The industrial operations on the product objects and the data generated in the operations are recorded and sent to the data cloud, and the transitions between industrial processes are recorded as well. For the manufacturer, the data of source materials can be accessed from the data cloud with their ID using IoT terminals. It processes the raw materials and save the collected data to the data cloud during the manufacturing. The finished products are labeled with IDs for the market to read and access the product data from the data cloud. Therefore, with the IDs and IoT pervasive monitoring, all collected data are available to the source supplier, manufacturer and market for further use. The end users may also be allowed to access the interested information.

This technical framework could potentially allow the data collection and sharing between the authorized business partners through the entire product lifecycle. The collected data could benefit the industrial operations and the system optimization.

\section{B. RFID Unique Product Localization and Identification}

For highly adaptive manufacturing systems, industrial operations are fulfilled by the smart machines automatically. A key prerequisite is to allow the machines to identify the product items in their workspaces. For RFID labelled products, it raises challenges to uniquely identify an item due to the multiple tag reading property of Ultra High Frequency (UHF) RFID.

In this investigation, the Received Signal Strength Indicator (RSSI) of RFID reading is utilized as the means for unique identification of product items. In theory, the RSSI reading is proportional to the biquadrate of the reciprocal of distance. The free-space propagation model can be expressed as follows:

$$
P_{r}=P_{t} \frac{G_{t}^{2} \lambda^{2} \sigma}{(4 \pi)^{3} d^{4}}
$$

In (1), $P_{t}$ and $P_{r}$ represent the emission power and received power of RFID reader, $G_{t}$ is the gain of reader antenna, $\lambda$ is the carrier wavelength, $\sigma$ is the Radar Cross Section (RCS) of tag, and $d$ is the distance between reader and tag. In reality, $G_{t}, \lambda$ and $\sigma$ are fix values for a set of RFID reader system and a specific type of tags. Thus, the distance $d$ between the reader and tags can be calculated by measuring $\mathrm{P}_{\mathrm{r}}$, which is usually expressed by RSSI $\left(P_{R}\right)$ defined as the ratio between the received power and reference power $\mathrm{P}$ (normally, $\mathrm{P}=1 \mathrm{~mW}$ ):
Suppose the reader antenna is in a fixed position, and let $\left(\mathrm{x}_{\mathrm{i}}\right.$, $\mathrm{y}_{\mathrm{i}}, \mathrm{z}_{\mathrm{i}}$ ) denote the position of tag $\mathrm{i}$, the RSSI can be presented by:

$$
P_{R}(i)=f\left(x_{i}, y_{i}, z_{i}\right)
$$

Normally, when the product items are moving forward in the manufacturing line, the $\mathrm{y}_{\mathrm{i}}$ and $\mathrm{z}_{\mathrm{i}}$ are fixed. Therefore,

$$
\begin{gathered}
P_{R}(i)=f\left(x_{i} \mid y=y_{0}, z=z_{0}\right) \\
x_{i}=f^{-1}\left(P_{R}(i), y_{0}, z_{0}\right)
\end{gathered}
$$

Since the industrial environment may introduce multi-path effect to the RF propagation, the linearity of the above relationship is not as good as ideal free-space environment. As RFID reader always recognize all the ambient RFID tags, it is an important step to filter the tags to determine the one that is located in the workspace of a machine. By installing RFID antennas in fixed positions in the feed-in tunnels of input/output crates, Algorithm 1, a threshold-based RFID unique detection method using a $\mathrm{P}_{\mathrm{R}}$-X pattern, is employed for the identification of moving RFID objects. By comparing the RSSIs of the $n$ RFIDs with the threshold, the one with the minimum distance in a defined error interval is considered the RFID which has just passed by the antenna. This radiation pattern based RFID filter provides a cost-effective solution to simultaneously identify and localize the moving input/output crates among the ambient RFID objects in real time. The real pattern that fits the practical industrial scene can be obtained conveniently using priori experimental data, and the pattern matching is more lightweight compared to peer RFID localization approaches.

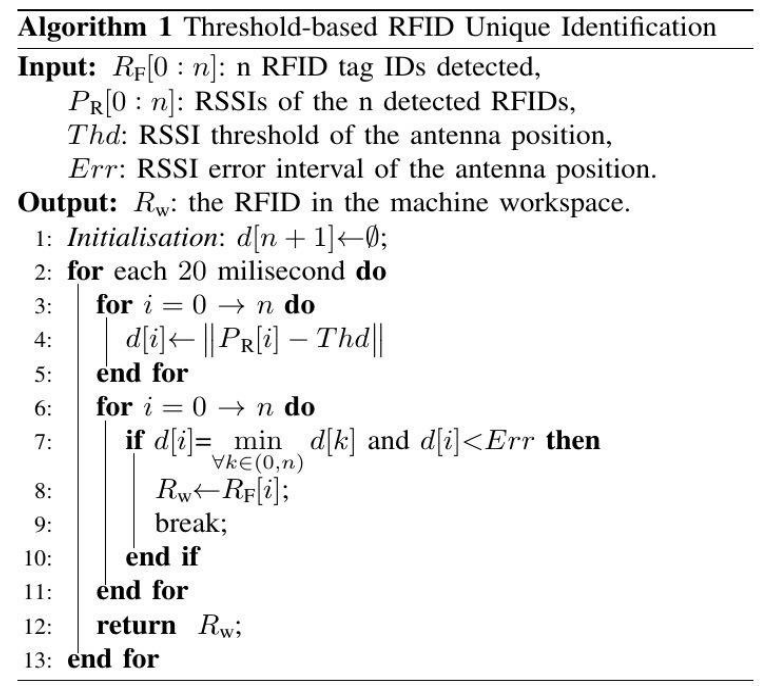




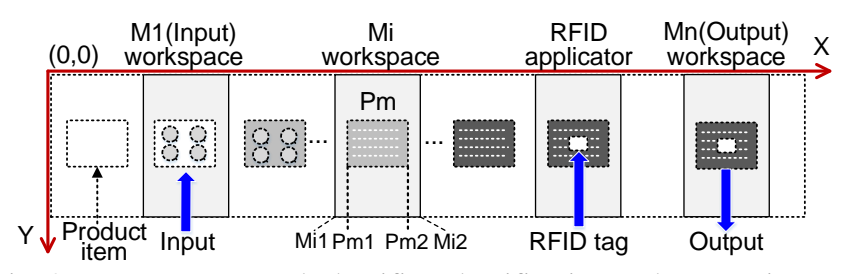

Fig. 3. UUID-RFID Dual Identifier Identification and Integration

\section{RFID and UUID Dual Identifier Online Data Integration}

For on-manufacturing-line industrial operations, the RFID tags can only be labeled on the products at a certain stage. The pre-RFID stages also need to identify the product items to build a connection with the later stages. Thus, a virtual UUID is applied for pre-RFID stage identification and integration.

As shown in Fig. 3, suppose the outside frame is the manufacturing line and small boxes inside are product items moving on the line through the workspaces of different machines. In the coordinate system, the X-direction locations of each machine $\left[\mathrm{XM}_{\mathrm{i} 1}, \mathrm{XM}_{\mathrm{i} 2}\right]$ is fixed. The position of a product item on the line can be measured with a precise encoder machine, which broadcasts the virtual UUIDs, the dimension of newly created product batch and the X-direction displacement.

To determine the product items in their workspace, each machine builds a buffer (BUF $[0, \ldots, \mathrm{m}-1])$ to accommodate the UUIDs and sizes of unprocessed items and calculate with Algorithm 2 to obtain the UUIDs. In Algorithm 2, the encoder data of new batches, each include 3 food packages, is put into a data buffer. The software continuously calculates the left and right edges of the front batch. If the left and right edges are both in the machine workspace, the product batch is considered the one in the workspace. The processed product items will be removed from the buffer. With this method, each machine can determine the virtual UUIDs of the product items in its workspace and register the data of manufacturing operations for integration before RFIDs are applied. Once an RFID is placed on a product item, the RFID applicator calculates the encoder position and determine the UUID of the item which is in its workspace and link it with the RFID to integrate the online generated data. The encoder position based virtual UUIDs allow the smart machines to determine the product IDs for data

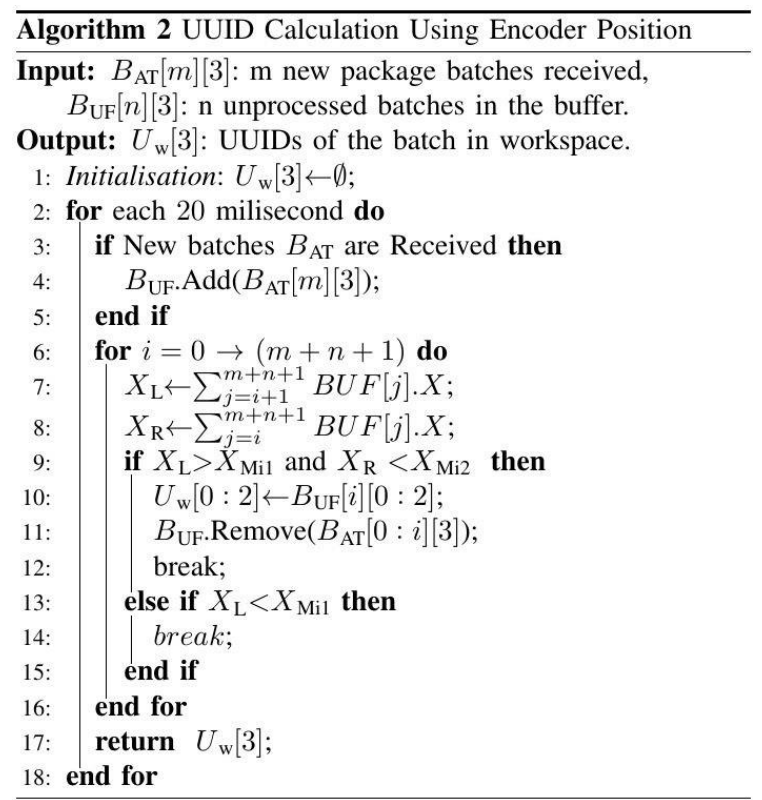

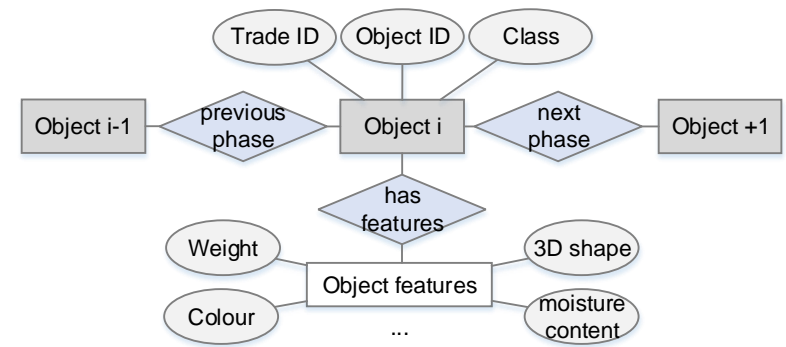

Fig. 4. An Object-centric Model of Product Data

registration in the early manufacturing stages before product labels are applied. The encoder position technique and virtual UUID-RFID dual-identifier technique have creatively bridged the gap between pre- and post-RFID manufacturing stages for product data integration. The combination of virtual UUIDs and physical RFIDs is a novel solution empowered by IoT techniques for on-manufacturing-line data integration of smart robotics based manufacturing applications.

\section{An Object-centric Model for Data Integration and Sharing}

To allow the product items and components to be identifiable through the lifecycle of production is a prerequisite of PLM, and the suitable models to describe the product data and operations are crucial technique issues. This section presents an object-centric modeling method for inter-phase data integration. 1) Object-centric product data modeling

To seamlessly combine the industrial processes, a product at any production phase is defined as an object. The objects can be integrated or processed to new objects at the later phases of production. As shown in Fig. 4, each object has an object ID, trade ID, class attributes, and the features of objects are defined by the class of objects. It also has the properties of previous and next phases object IDs to integrate with its previous and next phases. Therefore, the features of the objects at all production phases can be accessed through the links between object IDs, and the automatic generation of the links between objects can be achieved using the IoT sensing technologies like RFID.

2) Process Modeling for Inter-phase Data Integration

With the object-centric model and ID techniques, the data of a product item at all of its lifecycle phases could be integrated. As shown in Fig. 5, suppose there are $n$ phases in the manufacturing lifecycle, when the operations of object $i$ at phase $\mathrm{i}$ is completed, machines of phase $(\mathrm{i}+1)$ can obtain the data of phase $\mathrm{i}\left(\mathrm{p}_{\mathrm{i}}\right)$ by recognizing the trade ID $\left(\mathrm{TID}_{\mathrm{i}}\right)$ and object ID $\left(\mathrm{OID}_{\mathrm{i}}\right)$. Therefore, the data of $\mathrm{n}$ phases of a finished product can be accessed by the link of IDs, which can be expressed as:

$$
\begin{gathered}
T_{i-1}=\operatorname{Pr} \text { eviousTIDof }\left(T I D_{i}\right) \\
\text { OID }_{i-1}=\operatorname{Pr} \text { eviousOIDof }\left(\text { OID }_{i}\right) \\
\text { Feature }\left(p_{i}\right)=\text { FeatureofID }\left(\text { TID }_{i}, \text { OID }_{i}\right)
\end{gathered}
$$$$
\text { Feature }(\text { product })=\sum_{i=1}^{n} \text { FeatureofID }\left(\text { TID }_{i}, \text { OID }_{i}\right)
$$

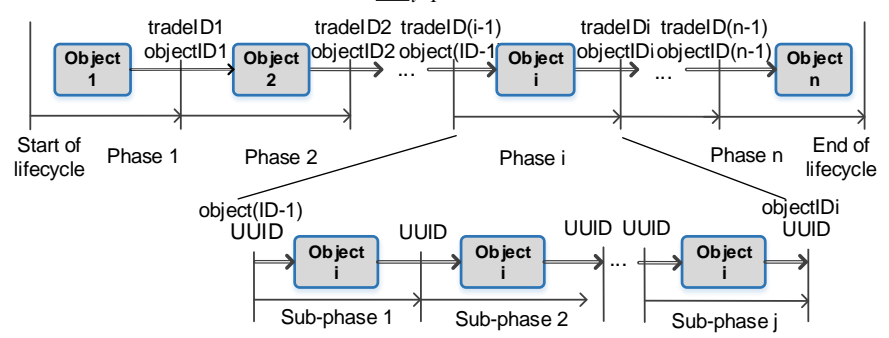

Fig. 5. Process Modeling for Inter-phase Data Integration 


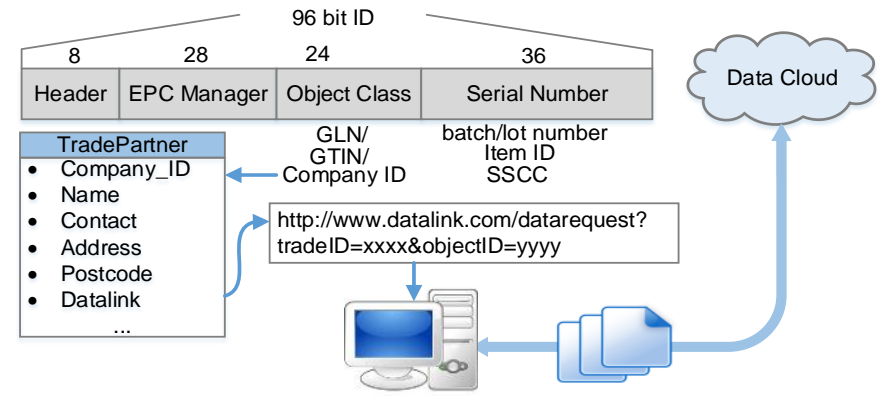

Fig. 6. ID Format Design for Data Integration and Sharing

For some on-manufacturing-line phases, it is not practical to place an RFID label or a $\mathrm{QR}$ code on the product items. Timing or mechanical encoding based virtual UUID could be used to identify the objects for data integration. When it is applicable to place a label, the label ID can be linked to the UUID to access the generated data, which is as shown in Fig. 5, the sub-phases 1 to $\mathrm{j}$ of phase $\mathrm{i}$. This is a well-suited solution for smart manufacturing, where industrial operations are carried out by collaborative automations of some interactive machines.

\section{E. RFID Encoding Method for Data Integration and Sharing}

RFID and QR code are the most suitable candidates for object identification, which have already been used in industry, logistics, and retail, etc. UHF RFID outperforms the other candidates in reading range, speed, data capacity, security, and therefore has gained wide acceptance. The Electronic Product Code (EPC) RFID standard developed by EPC Global in cooperation with Auto-ID Laboratories provides more details for object identification [26]. Normal EPC Class 1 Gen 2 tags have a 96-bit General Identification Number (GIN-96), which contains a header, an EPC manager, an object class and a serial number. Further schemes define more powerful codes, such as SGTIN-96, SSCC-96, and SGLN-96, where company prefix, item reference, etc. can be encoded.

In order to gain interoperable data access from trading partners in the manufacturing chain, a GIN-96 based data sharing method is designed as shown in Fig. 6. The trade ID and object ID are encoded in the object class and serial number sections of GIN-96 digits. The trade ID encoded in the object class section, which can be a company ID, a Global Location Number (GLN) of a trade partner, or a Global Trade Item Number (GTIN) of a product type, is linked to data access URL of the object saved in local database or the data cloud. With the data links and object IDs, the data of product objects can be accessed from the trade partners' data systems. This method simplifies the data sharing between trade partners by getting the link and object ID automatically via RFID reading. In addition
TABLE I

The HaRdware COMPONENTS OF RFID TRACEABILITY SySTEM

\begin{tabular}{lcc}
\hline \hline \multicolumn{1}{c}{ Device Type } & Device Model & Quantity \\
\hline RFID Reader & Alien ALR-9900+ & 1 \\
Antenna & Mobile Mark PN6-915 & 4 \\
Handheld reader & Alien ALH-9001 & 1 \\
RFID tag & Alien ALN-9629 & $/$ \\
RFID applicator & Label-Aire 3114NV Tamp-Blow & 1 \\
PC controller & Dell XPS 8500 Desktop & 1 \\
\hline \hline
\end{tabular}

to RFID, QR code can also encode the company ID and item ID for data sharing.

Focusing on the interoperable data integration and sharing in all production phases for lifecycle data management, this section has covered the key technical issues including product unique identification, online and offline data integration, object data and process modeling and flexible data sharing. The reference system architecture and technical solutions presented in this section are well-suited for IoT-enabled manufacturing.

\section{IMPLEMENTATIONS IN PICKNPACK PRACTICE}

To prove the feasibility of the proposed solutions discussed in Section III, this section presents the practice of the proposed technical solutions in PickNPack digital food manufacturing production line as a proof of concept demonstration.

\section{A. The PickNPack Food Manufacturing System}

PickNPack is a EU funded project which aims to integrate the emerging Information and Communication Technologies (ICT) and the state-of-the-art industrial devices such as robots, sensors, and controllers to build a flexible food manufacturing production line. For this rich sensing and communication system, data collection, data analysis, and data based optimization are the central concerns. This data-centric manufacturing flexibility is developed from the connected smart devices and the integration of IoT technologies for flexible data exchange and collaborative automations. The underlying ICT technologies have built a through-the-line information flow for product data management.

1) The structure of PickNPack manufacturing line

The structure of the PickNPack line is as shown in Fig. 7. A modularized design is applied to simplify the coupling of messages and events. There are 8 functional modules in the line:

- Thermoformer - a machine to produce shape and size adjustable food packages with plastic film.

- Pickrobot (input) - a robot to pick up raw food materials from input crates and place them in empty food packages.

- Quality Assessment and Sensing (QAS) - a sensor system to measure food quality with five different kinds of sensors, including RGB, 3D, hyperspectral, microwave, and X-ray.

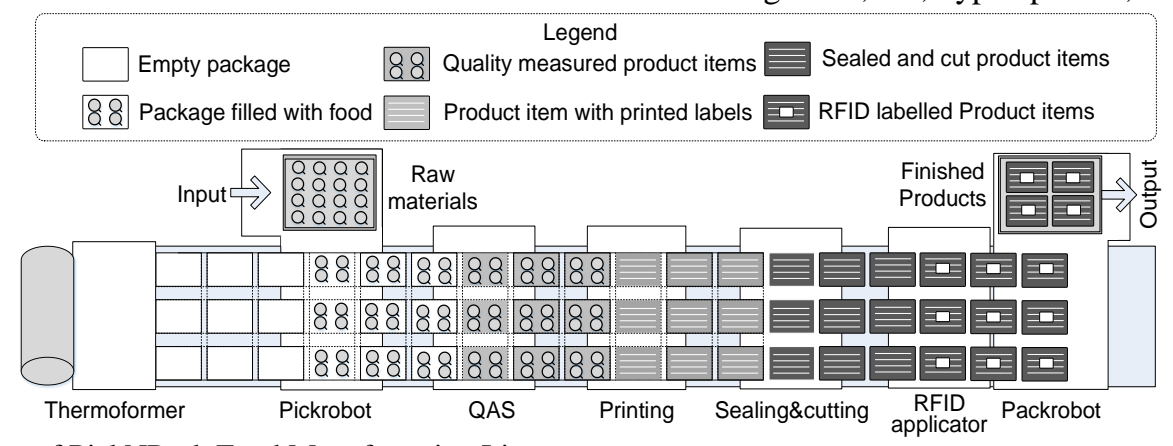

Fig. 7. Schematic Diagram of PickNPack Food Manufacturing Line 


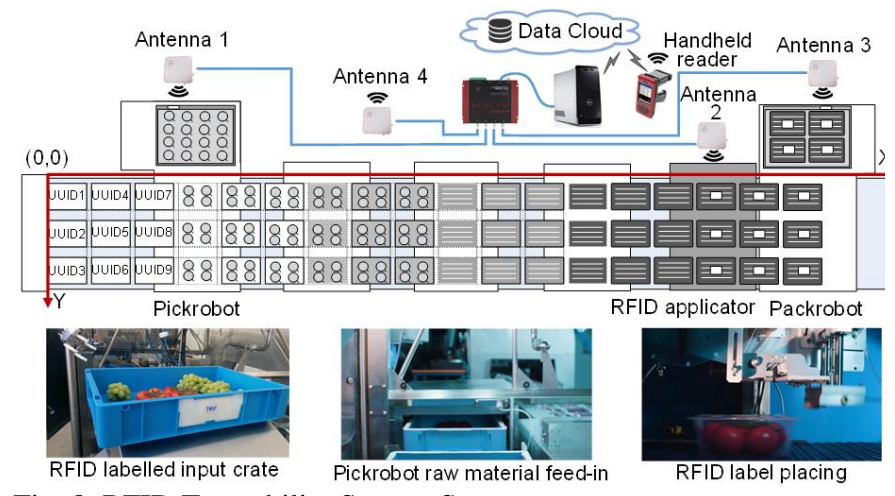

Fig. 8. RFID Traceability System Setup

- Printing - a label printer to print the particular information of the food products on the labels in real time.

- Laser sealing \& cutting - a laser system to seal the printed labels on the packages and cut them into separate items.

- Packrobot (output) - a robot to pick up the separated finished products and place them in output crates.

- Traceability - an RFID based process monitoring system for online data collection and sharing, and offline traceability.

- Cleaning - a robot module automatically flushing water to clean the whole system.

All the modules are connected to a local area network with Internet access. The product and production information is collected automatically by the machine modules using online virtual UUID for data integration. The RFID traceability system then integrates the online and offline phases by monitoring the input and output crates and RFID labelling of RFID applicator. 2) RFID traceability system setup

The RFID traceability system is set up to monitor the input and output crates and the product items RFID labelling, which consists of RFID reader, RFID antennas, RFID applicator, handheld RFID reader and PC controller as listed in TABLE 1.

The ALR-9900+ fixed reader is connected to a desktop running a custom-developed software to handle the data streaming of RFID readings. The software of both the desktop and the handheld reader are connected to the database for data storage and retrieval. A specific database model is developed to accommodate the product information and production process. As shown in Fig. 8, the antennas are installed as follows:

- Antenna1-in Pickrobot to detect RFID labelled input crates.

- Antenna2-in RFID applicator to detect the RFID placing.

- Antenna3-in Packrobot to detect RFID labelled output crates.

- Antenna4-for information tracing and item registration, etc.

Through the M2M messaging protocols, the RFID system can talk to the Pickrobot and Packrobot to notify them the crates' IDs in their workspaces. It can also receive the encoder position and calculate the UUIDs of product items that the RFID tags are placed on by the applicator.

\section{B. RSSI-based Object Localization and Identification}

For PickNPack manufacturing line, the product objects, such as product items, input crates and output crates need to be identified when they are moving or when they are among a couple of adjacent objects. To uniquely identify the RFID labelled objects with RFID readings, the pattern of RSSI reading of the moving product objects on the line is determined with the installed RFID devices, which is as shown in Fig. 9.
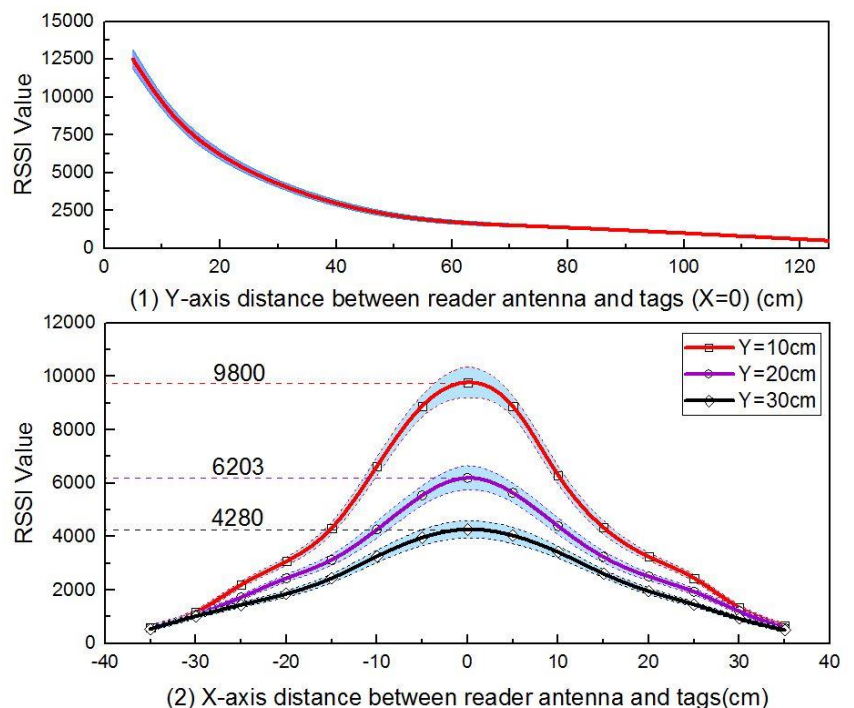

Fig. 9. Pattern of RSSI Reading in the RFID Moving Direction

Fig. 9(1) presents the RSSI reading of a tag moving away the antenna from $5 \mathrm{~cm}$ to $125 \mathrm{~cm}$, and Fig 9(2) describes the RSSI reading of an RFID labelled object moving on a conveyor belt in $\mathrm{X}$-axis from -35 to $35 \mathrm{~cm}$ away from the antenna with $\mathrm{Y}$-axis at $10 \mathrm{~cm}, 20 \mathrm{~cm}$ and $30 \mathrm{~cm}$. In the Figures, the wide colored line is the mean value and the light blue span is the error interval. The RFID labelled objects are recognized at the position where the antenna is placed $(\mathrm{X}=0)$ when they are moving toward $\mathrm{X}$ axis. The RSSI thresholds for Y-axis distance at 10, 20, and $30 \mathrm{~cm}$ are obtained using priori data which are indicated in Fig.9(2). With this experimental RSSI pattern, the RFID objects can be identified with Algorithm 1. With this method, the input Pickrobot can identify the input crate among plenty RFID objects when it goes to its workspace through the tunnel to integrate the source material information. The output Packrobot can identify the output crate among plenty RFID objects when it goes to its workspace through a tunnel to integrate the outgoing information of finished products for data integration.

Since the RSSI values vary with the reader radiation power, gain of antenna, RCS of tags and the influence of the ambient environment, the RSSI pattern should be recalibrated for specific antennas, tags categories and new installations. In PickNPack line, the motion track of input and output crates are shielded with metal covers to improve the quality of signals.

\section{RFID/UUID based Online and Offline Data Integration}

As described in Section III, the machines on the PickNPack line identifies the product items using UUIDs by calculating the encoder position which is circulated to the machines by the Thermoformer in the beginning of the line. Each machine on the line can timely identify the product item in its workspace and integrate the data generated in its operations with UUID.

As shown in Fig. 8, the integration of the online integrated data with offline data is achieved by combining the RFID with UUID in three intersections: input crates with raw food materials at Pickrobot, RFID labelling at RFID applicator, and output crates with finished products at Packrobot.

1) For the Pickrobot module, it is notified the valid raw material input crate identified by Antenna 1 of the RFID system. It also calculates the encoder information and identifies the UUID of the food package in its workspace. 


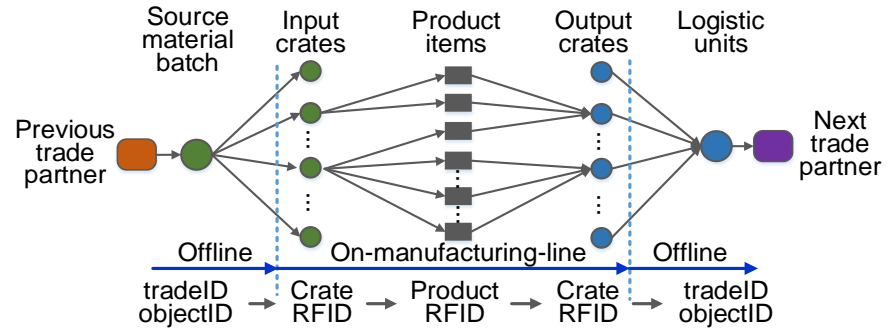

Fig.10. Workflow of Inter-phase Data Integration

When picking up raw food materials from the input crate and placing it into the empty package in its workspace, the Pickrobot registers the input crate RFID as the pre-phase object ID (source material RFID) property of the package. The item's UUID is linked with its raw material RFID.

2) For the RFID applicator, it calculates the encoder information and determines the UUID of the product item in its workspace. It then places an RFID tag on the product item and registers the RFID recognized by the Antenna 2 as the object ID (item RFID) property of the product item. The online UUID is linked with the product item RFID.

3) For the Packrobot module, it is notified the RFID of the valid output crate in its workspace recognized by Antenna 3. It calculates the encoder information and identifies the UUID of the product item in its workspace. When picking up the finished product item in its workspace and placing it in the output crate, it registers the output crate RFID as the next-phase object ID of the product item.

With the above method, the data generated by the functional modules can be collected and integrated with UUID. The combination of UUIDs with input crate and output crate RFIDs integrates the online generated data with the source material information and outgoing information. By combining the UUIDs and RFIDs with RFID applicator, the online generated data can be easily accessed with a machine readable approach.

\section{RFID Enabled Inter-Phase Data Integration and Sharing}

A critical step for lifecycle manufacturing data management is the data integration and sharing between different enterprises, including the source material provider, manufacturer and sales. The PickNPack line implements the inter-phase data integration and ubiquitous data access with the proposed process model and RFID/QR code encoding method as shown in Fig. 5 and Fig. 6.

When the source material batches are received by the manufacturer, they undergo a subdivision process which divides the batches with RFID labelled input crates. The input crate then registers the batch ID (RFID or QR code) as its previous-phase ID, consisting of the supplier ID and batch ID. The source materials in the input crates are made into product items on the manufacturing line. When finished product items are picked up and placed into the output crates, the items are then assembled to a logistic unit registering the unit ID as the next-phase ID (RFID or QR code), consisting of the outgoing company ID and unit ID. Since the trading partners have data link and permission of the other partners' data repository. The source provider, manufacturer and market can access the product information from their previous and next trading partners. The inter-phase data integration can be presented with Fig. 10. With the designed ID encoding format and phase integration method, a lifecycle data collection and sharing framework is therefore achieved. For a product item, the source information, production information and outgoing information can be accessed conveniently via RFID or QR code reading.

With the presented techniques, the PickNPack manufacturing line has achieved a lifecycle data collection, data integration and data sharing framework with the smart machines and IoT techniques. The interface of the PickNPack traceability software shown in Fig. 11 gives some data collected in a test of the system, which can demonstrate the functionalities achieved, including (1) modeling of the manufacturing industrial processes, (2) observation the state of peer machines on the manufacturing line, (3) collection of the

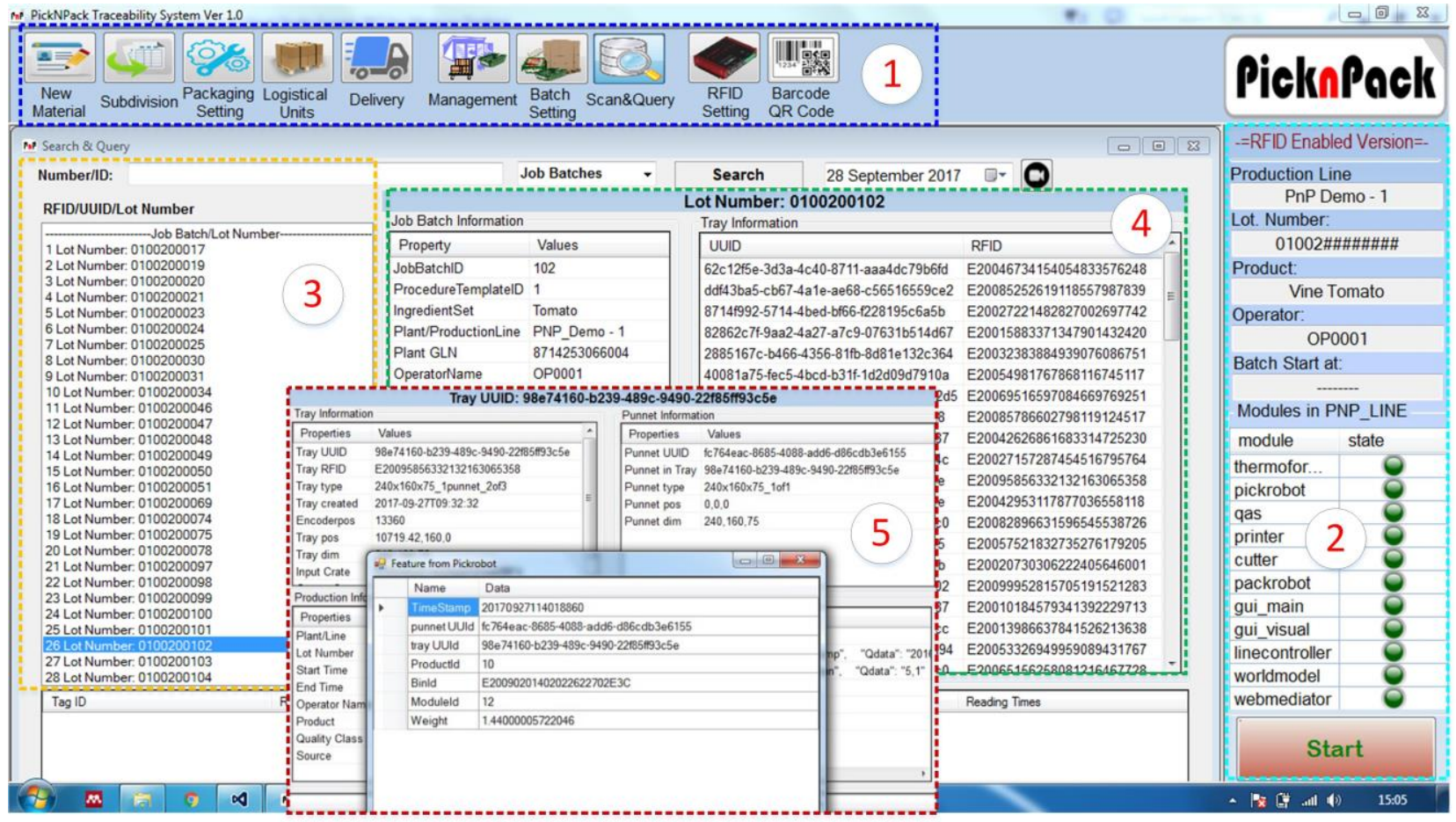

Fig.11. Interface of the PickNPack Information Traceability Software 
TABLE II

A SUMMARY OF PICKNPACK DATA INTEGRATION TECHNIQUES

\begin{tabular}{ll}
\hline \hline \multicolumn{1}{c}{ Functionalities } & \multicolumn{1}{c}{ Techniques } \\
\hline Pre-label identification/integration & Encoder position and virtual UUID \\
Pre- and post- RFID integration & Encoder position and RFID applicator \\
Inter-phase integration & RFID and object-centric model \\
Outgoing logistics data integration & RFID and object-centric model \\
Data sharing for trading phases & RFID encoding and object-centric model \\
\hline \hline
\end{tabular}

manufacturer's production batches, (4) collection of source information and registered product IDs of a production batch, (5) collection of the product data generated on the manufacturing line. Since the RFIDs of product items are combined with the corresponding UUIDs, the online generated data and offline data of the product items are integrated. The PickNPack manufacturing line was successfully demonstrated in Wageningen, Netherlands, and Holbeach, the UK, and the system on the demonstration site is as shown in Fig. 12.

\section{DISCUSSION}

IoT-based PLM, as a new manufacturing data management paradigm, is an important concept for future rich sensing smart manufacturing applications. This investigation provides a proof of concept demonstration of exploring IoT sensing and networking techniques to bridge the technical gap and build a boundary-less information flow for data integration and ubiquitous access through the entire manufacturing lifecycle.

The process modeling and information flow design for RFID based data management has been a widely studied area in the manufacturing industry. This investigation focuses on the exploration of IoT sensing and networking techniques and object-centric methods to build connectivity between onmanufacturing-line robot operation phases and offline logistics phases. TABLE II gives the key product identification and data integration techniques covering the PickNPack manufacturing phases and the corresponding functionalities achieved. Compared to some peer RFID based data management solutions which mainly focus on supply chain logistics information collection and distribution, the primary advantage of the presented solution lies in the dual identifier technique and object-centric methods which make it well-suited for the data registration and ubiquitous data access of smart machine based highly adaptive manufacturing applications, especially the prelabel unmanned manufacturing operations performed by smart machines. This allows the automatic and unobtrusive integration of raw material information, product information and outgoing information with minimized human involvement, which is challenging for the manufacturing operations when product labels are not applied.

The proposed technical solutions are proved to be feasible to deal with the technical challenges in smart manufacturing: (1) the RSSI pattern based unique identification technique can determine a moving RFID labelled item in multiple recognized items in real time, (2) the RFID and UUID dual identifier method can integrate the on-manufacturing-line pre-RFID stages with offline stages, (3) the object-centric model integrates the manufacturing phases with previous and next phases by registering their object IDs as properties, (4) the RFID and QR code encoding method allows to encode the data access information in the IDs for ubiquitous data sharing between trading partners. The implementations in PickNPack practice has demonstrated the feasibility and advantages.

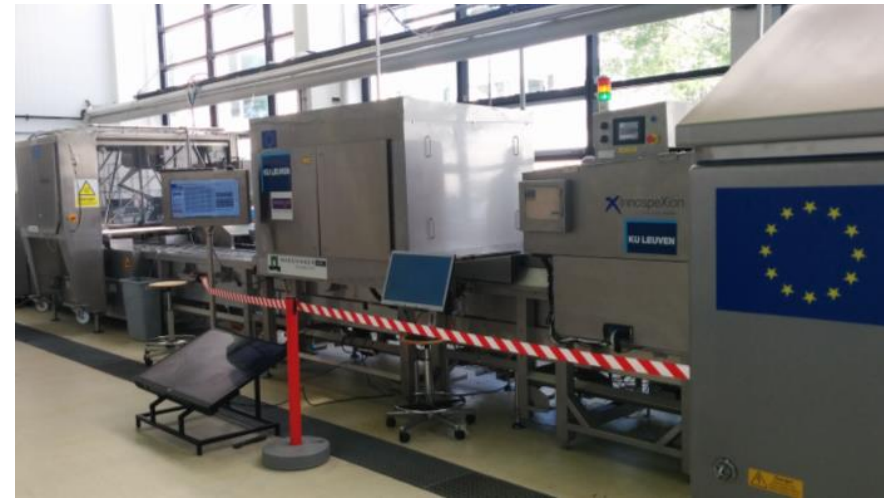

Fig.12. PickNPack Production Line in the Demonstration Site

The lifecycle data management requires the maintenance of a live information flow for the smart manufacturing machines, robotics, IoT sensing and computing terminals through all lifecycle phases. Therefore, the efficiency and reliability of network connection, messaging protocols and potential communication barriers are the main challenges to the success of the PLM information flow. In addition, since entities in the PLM are both data generators and data consumers, IoT collected data should be visible to authorized stakeholders in an interoperable way. The security and authentication for data access, data collection and data sharing are also critical issues.

\section{CONCLUSIONS}

This investigation provides a systematic PLM technical framework for smart manufacturing with IoT sensing and networking technologies and object-centric methods focusing on the product unique identification, dual-identifier online and offline data integration, object-centric modeling for inter-phase data integration and ubiquitous data access. The proposed solutions are a timely update for the data integration of emerging IoT-based smart manufacturing, which have demonstrated the feasibility and potentiality of IoT pervasive sensing and networking techniques in enhancing the data management and interactive automations of smart manufacturing. The designs and implementations are a successful practice to introduce IoT sensing and computing techniques to innovate practical manufacturing applications.

The proposed techniques have contributed to a boundary-less information flow with IoT sensing techniques covering onmanufacturing-line robotics operations before and after RFID labelling and offline phases data access between trading partners. The RSSI pattern based RFID filter provides a costeffective solution to simultaneously identify and localize the moving RFID objects in real time, which can be conveniently customized to fit the practical industrial scenes using priori experimental data. The encoder position based virtual UUID and RFID dual-identifier identification technique allows itemspecific identification and data integration of pre- and postRFID operations. The object-centric modeling then enables the inter-phase data integration with previous and next trading partners through the product lifecycle. The product objects then carry the information to the following lifecycle phases using the proposed RFID and QR code encoding method. The presented techniques and practices might be of interest to manufacturing researchers and practitioners for the innovation of their applications. 


\section{ACKNOWLEDGMENT}

The work presented in this paper is supported by the EU PickNPack project (Project code: 311987 ). The authors would thank all project partners for their cooperation in data communication and system integration.

\section{REFERENCES}

[1] S. Jeong, W. Na, J. Kim, S. Cho, "Internet of Things for Smart Manufacturing Systems: Trust Issues in Resource Allocation," IEEE Internet of Things Journal, 2018. DOI: 10.1109/JIOT.2018.2814063.

[2] J. Wan, B. Chen, M. Imran, et al., "Toward Dynamic Resources Management for IoT-Based Manufacturing, "IEEE Communications Magazine, vol. 56, no. 2, pp.52-59, 2018.

[3] C. Yang, W. Shen, X. Wang, "The Internet of Things in Manufacturing: Key Issues and Potential Applications," IEEE System, Man, and Cybernetics Magazine, vol. 4, no. 1, pp. 6-15, 2018.

[4] Z. Bi, L. Da Xu, and C. Wang, "A Survey on Internet of Things From Industrial Market Perspective," IEEE Transactions on Industrial Informatics, vol. 10, no. 2, pp. 1537-1546, 2014.

[5] M. Weyrich and C. Ebert, "Reference Architectures for the Internet of Things," IEEE Software, vol. 33, no. 1, pp. 112-116, 2016.

[6] X. Li, D. Li, J. Wan, C. Liu, and M. Imran, "Adaptive Transmission Optimization in SDN-based Industrial Internet of Things with Edge Computing," Internet of Things Journal, vol. 5, no. 3, pp. 1351-1360, 2018.

[7] F. Ameri and D. Dutta, "Product Lifecycle Management: Closing the Knowledge Loops," Computer-Aided Design and Applications, vol. 2, no. 5, pp. 577-590, 2005.

[8] H. B. Jun, D. Kiritsis, and P. Xirouchakis, "Research Issues on Closedloop PLM," Computers in Industry, vol. 58, no. 8-9, pp. 855-868, 2007.

[9] L. Xu, W. He, and S. Li, "Internet of Things in Industries: A Survey," IEEE Transactions on Industrial Informatics, vol. 10, no. 4, pp. 22332242, 2014.

[10] F. Tao, Y. Cheng, L. D. Xu, L. Zhang, B. H. Li, "CCIoT-CMfg: Cloud Computing and Internet of Things-Based Cloud Manufacturing Service System," IEEE Transactions on Industrial Informatics, vol. 10 no. 2, pp. 1435-1442, 2014.

[11]K. Framling, S. Kubler, and A. Buda, "Universal Messaging Standards for the IoT from a Lifecycle Management Perspective," IEEE Internet of Things Journal, vol. 1, no. 4, pp. 319-327, 2014.

[12]A. Bradley, "Manufacturing Connectivity and Data Integration," Ind. Week/IW, vol. 264, no. 2, pp. 2-7, 2015.

[13]H. Cao, P. Folan, J. Mascolo, and J. Browne, "RFID in Product Lifecycle Management: A case in the Automotive Industry," Internationa Journal of Computer Integrated Manufacturing, vol. 22, no. 7, pp. 616-637, 2009.

[14] F. Bibi, C. Guillaume, N. Gontard, B. Sorli, "A review: RFID technology having sensing aptitudes for food industry and their contribution to tracking and monitoring of food products," Trends in Food Sciences \& Technologies, vol. 62, pp. 91-103, 2017.

[15]M. Glaab, W. Fuhrmann, J. Wietzke, and B. Ghita, "Toward Enhanced Data Exchange Capabilities for the oneM2M Service Platform," IEEE Communications. Magazine, vol. 53, no. 12, pp. 42-50, 2015.

[16]M. P. Papazoglou, W. J. Van Den Heuvel, and J. E. Mascolo, “A Reference Architecture and Knowledge-based Structures for Smart Manufacturing Networks," IEEE Software, vol. 32, no. 3, pp. 61-69, 2015.

[17]K. Främling, J. Holmström, J. Loukkola, J. Nyman, and A. Kaustell, "Sustainable PLM through Intelligent Products," Engineering Applications of Artifical Intelligence, vol. 26, no. 2, pp. 789-799, 2013.

[18]D. Kiritsis, "Closed-loop PLM for Intelligent Products in the Era of the Internet of Things," Computer-Aided Design, vol. 43, no. 5, pp. 479-501, 2011.

[19]K. Främling, M. Harrison, J. Brusey, and J. Petrow, "Requirements on Unique Identifiers for Managing Product Lifecycle Information: Comparison of Alternative Approaches," International Journal of Computer Integrated Manufacturing, vol. 20, no. 7, pp. 715-726, 2007.
[20]T. Kamigaki, "Object-oriented RFID with IoT: A Design Concept of Information Systems in Manufacturing," Electronics (Switzerland), vol. 6, no. 14. pp. 1-11, 2017.

[21]Z. Meng, Z. Wu, C. Muvianto, and J. Gray, "A Data-Oriented M2M Messaging Mechanism for Industrial IoT Applications," IEEE Internet of Things Journal, vol. 4, no. 1, pp. 236-246, 2016.

[22]H. Cai, L. Xu, B. Xu, C. Xie, S. Qin, and L. Jiang, "IOT-based Configurable Information Service Platform for Product Lifecycle Management," IEEE Transactions on Industrial Informatics, vol. 10, no. 2, pp. 1558-1567, 2014.

[23]S. Rachuri, E. Subrahmanian, A. Bouras, S. J. Fenves, S. Foufou, and R. D. Sriram, "Information Sharing and Exchange in the Context of Product Lifecycle Management: Role of Standards," Computer-Aided Design, vol. 40, no. 7, pp. 789-800, 2008.

[24]R. Sudarsan, S. J. Fenves, R. D. Sriram, and F. Wang, "A product Information Modeling Framework for Product Lifecycle Management," Computer-Aided Design, vol. 37, no. 13, pp. 1399-1411, 2005.

[25] K. Yang, D. Forte, M. Tehranipoor, "ReSC: An RFID-Enabled Solution for Defending IoT Supply Chain," ACM Transactions on Design Automation of Electronic Systems, vol. 23, no. 3, pp. 1-27, 2018.

[26]Y. Xiao, S. Yu, K. Wu, Q. Ni, C. Janecek, and J. Nordstad, "Radio Frequency Identification: Technologies, Applications, and Research Issues," Wireless Communication and Mobile Computing, vol. 7, no. 4, pp. 457-472, 2007.

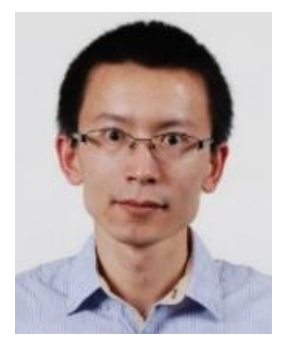

Zhaozong Meng (M'16) received his BSc and MSc degrees on Instrumentation and Measurement from Sichuan University, Chengdu, China, in 2006, and Beihang University, Beijing, China, in 2009. He received the $\mathrm{PhD}$ degree on computer science from University of Huddersfield, UK, in 2014. He then joined the University of Manchester, the UK as a research associate in June 2014. His research interests include instrumentation and measurement, novel sensing and computing networks, M2M communications and Internet of Things Techniques and systems.

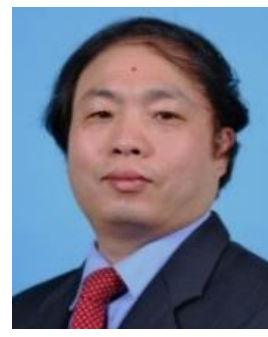

Zhipeng Wu (M'95-SM'05) obtained his BSc degree on Radio Technology from the Northeast University, China in 1983, and PhD degree on Antennas and Propagation from the University of Birmingham in 1988. He was also awarded a DEng degree by the University of Birmingham in 2003. During 1988-1991, he was appointed as a Research Fellow at the University of Birmingham. In 1992, he joined UMIST as a lecturer. He now severs as a professor of antenna and propagation at the University of Manchester. His research interests include microwave sensors, RF and microwave tomography, WSN, IoT, and techniques and systems for industrial applications, etc.

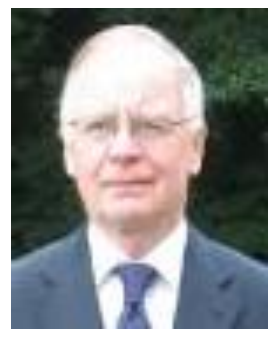

John Gray received his BSc degree on electrical engineering and MSc on semiconductor electronics from Queens University Belfast, Belfast, UK in 1959 and 1961 respectively, and the $\mathrm{PhD}$ degree in control systems from The University of Manchester, Manchester, UK in 1967. He was appointed a Lecturer in electrical engineering with University of Manchester. In 1979, he was appointed as the Chair of Control Engineering, University of Salford, Greater Manchester, UK He is currently a Professor in robotics and systems engineering with The University of Manchester. His research interests include robotics, automation in food manufacturing, and digital manufacturing. 